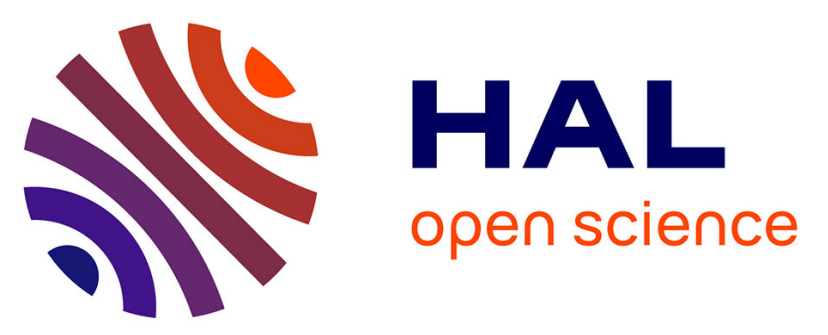

\title{
Electronic and structural properties of interstitial titanium in crystalline silicon from first-principles simulations
}

Gabriela Herrero-Saboya, Layla Martin-Samos, Anne Hémeryck, Denis Rideau, Nicolas Richard

\section{To cite this version:}

Gabriela Herrero-Saboya, Layla Martin-Samos, Anne Hémeryck, Denis Rideau, Nicolas Richard. Electronic and structural properties of interstitial titanium in crystalline silicon from first-principles simulations. 2019 International Conference on Simulation of Semiconductor Processes and Devices (SISPAD), Sep 2019, Udine, Italy. pp.1-4, 10.1109/SISPAD.2019.8870382 . hal-02338691

\section{HAL Id: hal-02338691 \\ https://hal.laas.fr/hal-02338691}

Submitted on 30 Oct 2019

HAL is a multi-disciplinary open access archive for the deposit and dissemination of scientific research documents, whether they are published or not. The documents may come from teaching and research institutions in France or abroad, or from public or private research centers.
L'archive ouverte pluridisciplinaire HAL, est destinée au dépôt et à la diffusion de documents scientifiques de niveau recherche, publiés ou non, émanant des établissements d'enseignement et de recherche français ou étrangers, des laboratoires publics ou privés. 


\section{Electronic and structural properties of interstitial titanium in crystalline silicon from first-principles simulations}

\author{
Gabriela Herrero-Saboya \\ CEA, DAM, DIF \\ Arpajon, France \\ $L A A S-C N R S$ \\ Toulouse, France \\ gabriela.herrerosaboya@cea.fr \\ Denis Rideau \\ STMicroelectronics \\ Crolles, France \\ denis.rideau@st.fr
}

\author{
Layla Martin-Samos \\ CNR-IOM / Democritos National \\ Simulation Center, c/o SISSA \\ Trieste, Italy \\ mrsamos@iom.cnr.it
}

Nicolas Richard

CEA, DAM, DIF

Arpajon, France

nicolas.richard@cea.fr

\author{
Anne Hemeryck \\ $L A A S-C N R S$, \\ Université de Toulouse \\ Toulouse, France \\ anne.hemeryck@laas.fr
}

\begin{abstract}
We demonstrate the presence of small JahnTeller distortions for interstitial titanium in silicon at different charge states by performing ground state DFT calculations. We prove the existence of three charged transition levels within the band gap by using a non-empirical parameter-free approach, based on the GW approximation, in agreement with DLTS measurements.
\end{abstract}

Keywords—titanium, impurity, point defects, silicon, firstprinciples

\section{INTRODUCTION}

Point-like defects are known to introduce trap levels in the semiconductor band gap allowing carrier recombination and charge capture through non-radiative transitions, modifying/degrading the performance of microelectronic devices. The accurate prediction of point defects is, therefore, a major challenge in microelectronics that has been and is still addressed by both theory and experiments.

Among all defects, Transitions Metals (TMs) impurities are common contaminants in semiconductors. In particular, titanium is believed to cause uncontrolled avalanches in Single-Photon Avalanche Diodes (SPAD). These diodes, by working at the limit of breakdown, are able to detect single photons arriving to the device, triggering an avalanche of carriers. If impurities such as titanium are present, even at low concentrations after the fabrication process, carriers can be generated thermally due to the presence of deep levels and therefore, avalanche processes can be triggered even without external stimulus, i.e. in the dark. We believe that a first approach to the problem is to characterize such traps levels theoretically to correctly identify their experimental signature. Understanding the atomic-scale origin of Tirelated activity is an enabling stage to design mitigation strategies.

TMs impurities in silicon, such as Ti and V, were first characterized by Electronic Paramagnetic Resonance (EPR) $[1,2]$. The variation of spin between TMs and different charge states was explained through the modification of the electronic structure of $3 \mathrm{~d}$ metals embedded in silicon, described by the Ludwig-Woodbury model $[1,2]$. According to such EPR-based model, the initial [Ar] $4 \mathrm{~s}^{2} 3 \mathrm{~d}^{\mathrm{n}}$ electronic configuration would be replaced by a $3 \mathrm{~d}^{\mathrm{n}+2}$ structure, where the $3 \mathrm{~d}$ levels are splitted into the so-called $t 2$ and $e$ levels with a multiplicity of 6 and 4 respectively (see Fig. 1). The measured spin value was first estimated by filling these electronic levels following Hund's rule. First-principles studies [3] reported, however, deviations from this empirical rule for both $\mathrm{Ti}$ and $\mathrm{V}$, after obtaining spin values of 1 and $1 / 2$ for Ti (V) at charge states $0(-1)$ and $-1(-2)$, instead of 2 and $5 / 2$ as predicted by the Ludwig-Woodbury model. They also predicted a $3 / 2$ spin value for Ti at charge +1 and for $\mathrm{V}$ at charge +2 , in agreement with EPR measurements performed by D. A. Wezep and coworkers [4,5]. Higher charge states have, however, never been observed experimentally. Besides the magnetic properties of TMs in silicon, EPR studies also concluded that both $\mathrm{Ti}^{1+}$ and $\mathrm{V}^{2+}$ are found in tetrahedral interstitial positions in the lattice with full Td symmetry.

Regarding the electronic properties of TMs, Deep Level Transient Spectroscopy (DLTS) studies [6-11] commonly agree on the presence of three Charge Transition Levels (CTLs) for interstitial Ti within the band gap; an acceptor level at $\mathrm{E}_{\mathrm{C}}-0.09 \mathrm{eV}$, a donor level in the range of $\mathrm{E}_{\mathrm{V}}+$ 0.87-0.92 eV, and a double donor level at $\mathrm{E}_{\mathrm{V}}+0.25-0.32$ $\mathrm{eV}$. References $[6,7,9]$ also reported intermediate band gap levels between $\mathrm{E}_{\mathrm{V}}+0.51 \mathrm{eV}$ and $\mathrm{E}_{\mathrm{V}}+0.55 \mathrm{eV}$. CTLs were also predicted within the framework of Density Functional Theory (DFT) [3,12-14] and the parametrized method $\mathrm{DFT}+\mathrm{U}$ [15], in fair agreement with most experimental measurements. In the context of standard DFT, however, there is no grounded theoretical foundation for an accurate prediction of CTLs. Indeed, as it is well known, DFT is not Self-Interaction free and it is not piecewise linear against adding/removing an integer charge number.

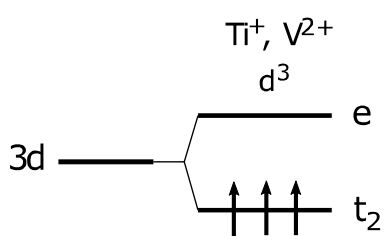

Fig. 1. Electronic configuration of interstitial Ti at charge $+1\left(\mathrm{Ti}^{1+}\right)$ and interstitial vanadium at charge $+2\left(\mathrm{~V}^{2+}\right)$. 
In this work, we discuss the properties of interstitial titanium at the atomic scale by performing first-principles simulations within DFT, for the ground state characterization, and Many-Body Perturbation Theory within the GW approximation for CTLs. The followed approach [16] is parameter-free and non-empirical, allowing an unbiased comparison with experimental measurements.

\section{METHODOLOGY}

Structural and magnetic properties are obtained by means of the Density Functional Theory (DFT) as implemented in the ABINIT code [17]. In order to avoid spurious defectdefect interactions due to the Periodic Boundary Conditions (PBC), a silicon supercell containing $3 \times 3 \times 3$ unit cells $(216$ atoms) and one k-point (gamma) are employed. The calculations are performed using the norm-conserving pseudopotential ONCVPSP [18] and the Perdew-BurkeErnserhof exchange-correlation functional [19]. A $30 \mathrm{Ha}$ energy cutoff for the plane wave basis set is found to give converged results. The defect geometries at different charge states are obtained by the BFGS algorithm, with a stopping force criteria of $1 \mathrm{meV} / \AA$. Furthermore, we are able to describe the magnetic properties of the system by performing spin-unrestricted calculations.

Many-body corrections are computed on top of the KohnSham energies within the GW method $\left(\mathrm{G}_{0} \mathrm{~W}_{0}\right.$ as implemented in the ABINIT code [17]) in order to obtain the defect band structure correctly. We employ the Godby-Needs plasmon-pole model and a cutoff energy of $3 \mathrm{Ha}$ to describe the dielectric matrix. In order to assure convergence of the GW exchange-correlation self-energy, we use a very large ratio of 10:1 empty bands versus occupied bands.

\section{RESULTS}

The capture or the release of a free carrier by a trap induces a structural reorganization of the defect. We therefore start this section by briefly discussing ground states properties of interstitial $\mathrm{Ti}$ and $\mathrm{V}$ at different charge states. We then present the electronic properties of the defects, focusing on the calculation of thermodynamic transition levels and the comparison with experimental evidence.

\section{A. Structural and magnetic properties}

Our ground state calculations also predict spin values of $1 / 2,1$ and $3 / 2$ for $\mathrm{Ti}(\mathrm{V})$ at charge states $-1(0), 0(+1)$ and +1 $(+2)$, confirming the break of Hund's rule and the preference for lower spin values. We also obtained the full $\mathrm{Td}$ symmetric interstitial configuration, characterized by four equivalent distances between the TM and the host $\mathrm{Si}$ atom (TM-Si) of $2.48 \AA$ for $\mathrm{Ti}^{1+}$ and $2.46 \AA$ for $\mathrm{V}^{2+}$.

Point-defects in semiconductors often present the socalled Jahn-Teller distortions when varying the trapped charge. The existence of such distortions in the case of interstitial Ti and $\mathrm{V}$ was hypothesized previously by $[12,14]$ but it was never reported as a result. Therefore, in contrast with previous theoretical results, we observed small JahnTeller distortions (see Fig. 2), in the order of $0.03 \AA$. In the case of $\mathrm{Ti}^{0}$ and $\mathrm{V}^{+}$, we obtained a flatted tetrahedron, constituted by one short distance TM-Si of $2.45 \AA$ and 2.42 $\AA$ respectively, against three longer distances of $2.48 \AA$ and $2.46 \AA$. $\mathrm{Ti}^{1-}$ and $\mathrm{V}^{0}$, on the contrary, present a slightly elongated configuration, characterized by a long distance of $2.49 \AA$ and $2.47 \AA$ and three shorter distances of $2.45 \AA$ and
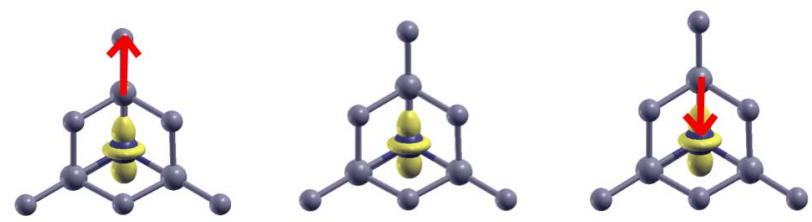

Fig. 2. Jahn-Teller distortions for interstitial $\mathrm{Ti}$ in silicon. Red arrows represent the geometry of the elongated (left) and flatted (right) tetrahedron. In yellow, one of the defect $t 2$ localized electronic states.

$2.42 \AA$ respectively. The origin of these slight changes in the defect geometry is the orientation of the $t 2$ states along the bonding directions as shown in figure 2 . The capture of a free carrier starting from a symmetric $\mathrm{Td}$ configuration would, therefore, lead to a distorted flatted geometry as the electron is allocated in such $t 2$ state. Small Jahn-Teller distortions of this order of magnitude should be observed in EPR signals; however, as it was already mentioned above, such signals have not been reported.

\section{B. Electronic properties}

Even though DFT correctly describes structural and magnetic properties of defects, the discontinuity of the exchange-correlation functional and the Self-Interaction contribution constitute the origin of the well-known DFT band gap problem, making the description of electronic properties a difficult task. In the present work, for example, we estimate the value of the silicon forbidden gap to be 0.75 $\mathrm{eV}$ in DFT, instead of the measured gap equal to $1.2 \mathrm{eV}$. A better treatment of the electronic correlation is possible by means of Many-Body Perturbation Theory within the GW approximation. The corrected GW energies or quasiparticle energies correspond to ionization potentials, IP, and/or electronic affinities, EA; and therefore, the silicon band gap can be estimated as, $\mathrm{EA}_{\text {bulk }}-\mathrm{IP}_{\text {bulk }}=1.18 \mathrm{eV}$. By combining both DFT and GW calculations we are able to compute the quasiparticle band structure of the defect, identifying the trapped states by studying the localization properties of the DFT wavefunctions (see Fig. 3).

In figure 3 we show the quasiparticle band structure for interstitial $\mathrm{Ti}+$. Even though three peaks appear within the silicon forbidden gap, only two of them can be considered as deep defect levels due to the spatial localization of their electronic wavefunctions (states $t 2$ and $e$ in Fig. 3, with a multiplicity of 6 and 4 respectively). The non-labeled state is actually part of the conduction band, since it presents similar delocalization properties as the ones of pure silicon. The small difference in energy is due to the structural distortions around the impurity and it is often referred to as the band gap narrowing effect. From the common structural and magnetic properties presented by interstitial titanium and vanadium, it is not surprising that, for the same number of trapped electrons (e. g. electronic occupation $3, \mathrm{~d}^{3}$, for $\mathrm{Ti}^{1+}$ and $\mathrm{V}^{2+}$ ), we obtain equivalent quasiparticle band structures (a triply occupied $t 2$ state). In the case of $\mathrm{Ti}^{1+}$, the occupied $t 2$ state is located at a distance of $\mathrm{IP}_{\mathrm{t} 2}-\mathrm{IP}_{\text {bulk }}=0.41 \mathrm{eV}$ from the top of the valence band (see Fig. 3). The occupied $t 2$ state for $\mathrm{V}^{2+}$, on the other hand, is located within the bulk valence band, in consistency with the absence of a triple donor state, $(++/+++)$, in the measured CTLs [20, 21]. 

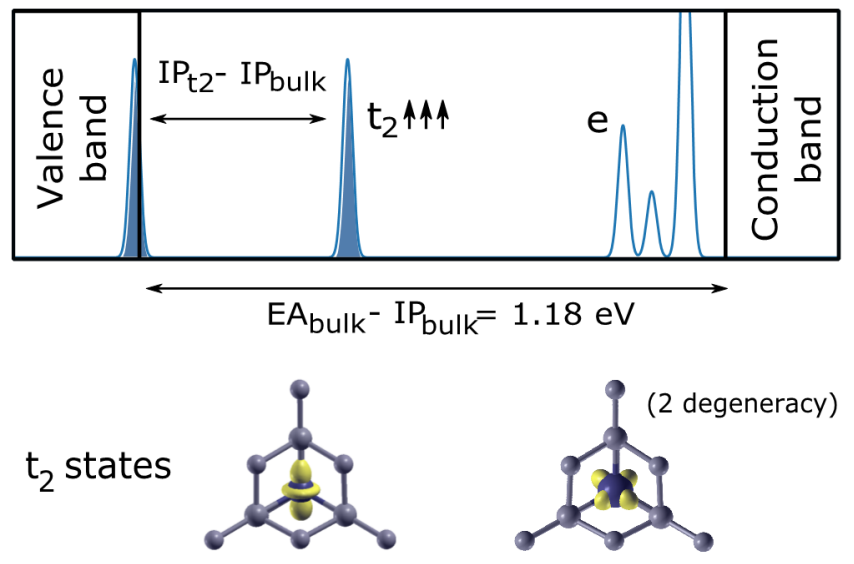

Fig. 3. (Above) Spin-up projection of the quasiparticle density of states for $\mathrm{Ti}^{1+}$. Occupied states are colored and the valence and conduction band are shown for the Si-bulk are shown. An electrostatic correction is applied to the GW energies according to [24]. (Below) Filled electronic defect states, called $t 2$ states.

The Charge Transition Levels (CTLs) are defined as the change in total energy between two different charge states at their corresponding relaxed geometries, with respect to the top of the valence band. Such energy difference can easily be computed from DFT ground state calculations; however, the estimated CTLs would suffer from the already mentioned band gap problem. A common strategy to avoid such underestimation [12-15] is the use of an empirical method, known as the Marker Method [22]. The idea of the method is to shift the computed CTLs by the absolute error determined for a "well characterized" defect; the error is defined as the difference between the calculated CTLs and the experimental one. In the present work, CTLs are computed using the nonempirical DFT-GW combine approach [16]. The gain/loose in energy when capturing/releasing an electron is accounted by the GW eigenvalues (first electronic affinity for capture and first ionization potential for releasing). Indeed, by definition, GW eigenvalues are the excitation energies $(\mathrm{N}+1 / \mathrm{N}-1)$ of an interacting $\mathrm{N}$-electron system. The energy exchange during structural reorganization can be safely described by DFT since no change in the particle number occurs in such process. The CTLs are therefore computed as follows,

$$
\mathrm{E}(+/ 0)=\mathrm{IP}_{\text {defect }}^{0}-\Delta \mathrm{E}_{\text {relax }}-\mathrm{IP}_{\text {bulk }}
$$

where $\mathrm{E}(+/ 0)$ represents a single donor level, $\mathrm{IP}_{\text {defect }}^{0}$ is the first IP computed from charge zero and $\Delta \mathrm{E}_{\text {relax }}$ the energy difference between the ground state geometries at charges 0 and +1 . Furthermore, as it was investigated by [23], the calculation of double acceptor/donor levels is not straight forward because of strong electrostatic interactions between localized charges in neighboring cells (we recall the use of $\mathrm{PBC}$ ). Even though GW corrections are short ranged, Hartree and ion-electron contributions coming from the DFT eigenvalues are subjected to this spurious electrostatic interaction. In the present work, we use the monopole correction scheme, proposed by G. Makov and M. C. Payne [24], to shift our $\mathrm{IP}^{+} / \mathrm{EA}^{+}$in order to calculate the double donor level, $(+/++)$. The obtained CTLs are shown in Table $\mathrm{I}$, as well as previous reported values $[13,15]$ and experimental evidence [6-11].
TABLE I. Charge Transition Levels (CTLs) for interstitial Ti. The acceptor level is computed from the conduction band, $\mathrm{E}_{\mathrm{C}}-\mathrm{E}(0 /-)$, whereas the donor levels are described from the top of the valence band $E_{V}+E(+/ 0)$. All values are given in $\mathrm{eV}$.

\begin{tabular}{ccccc}
\hline $\begin{array}{c}\text { Transition } \\
\text { level }\end{array}$ & DFT@GW & DFT [13] & DFT+U [15] & DLTS [6-11] \\
\hline \hline $\mathrm{E}(0 /-)$ & 0.19 & 0.14 & 0.05 & 0.09 \\
\hline $\mathrm{E}(+/ 0)$ & 0.82 & 0.51 & 0.98 & $0.87-0.92$ \\
\hline $\mathrm{E}(+/++)$ & 0.45 & 0.10 & 0.23 & $0.25-0.32$ \\
\hline \hline
\end{tabular}

Previous references employed an empirical method to shift their computed CTLs [13,15], since by using a pure $a b$ initio approach based on DFT, the calculated CTLs could not be predicted above the underestimated band gap (as in the case of $E(0 /-)$ in references $[13,15]$ and $E(+/ 0)$ for [15]). As we already showed in Fig. 3, by computing GW corrections on top of DFT, the silicon band gap is in very good agreement with experiments, and therefore, no empirical scheme is needed to shift our values. Furthermore, reference [15] relies not only on an empirical correction, but also on a parametrized first-principles calculation: the $\mathrm{DFT}+\mathrm{U}$ method. The basic idea behind this method is to describe the strong interaction between localized electrons by an additional Hubbard-like term, characterized by two parameters $U$ and J. LDA $+U$ allows for a recovery of the piecewise linearity for adding/removing electrons from strongly localized frontier orbitals (Top of Valence Band and/or Bottom of Conduction Band). Depending on the CTLs the $U / J$ value might vary significantly (see for instance figure 2 from [15]). Large variations are, however, difficult to meaningful interpret and theoretically ground.

It was established that theoretical CTLs could be assigned to DLTS activation energies [25]. Previous DFT studies already linked the acceptor level to the E40 characteristic peak, the donor level with the E150 signal, and the double donor level with the H180 peak. Due to the proximity of our double donor level to the mid-gap region, we considered the existence of a forth CTL within the values of $\mathrm{Ev}+0.51-0.55 \mathrm{eV}$ as suggested by DLTS experiences $[6,7,9]$. In order to clarify this point, CTLs for interstitial vanadium were also computed and compared to DLTS measurements as shown in Fig. 4. As already discussed above, interstitial vanadium presents a similar electronic structure as $\mathrm{Ti}$ (splitting and filling of the $3 \mathrm{~d}$ states). However, due to the difference in atomic number and therefore, electrostatic interaction with the nuclei, levels corresponding to the same change in electronic occupation differ for these two systems. This is the case, for example, of the $\mathrm{d}^{3} / \mathrm{d}^{4}$ transition, which corresponds to the CTL $(+/ 0)$ for Ti and (-/0) for V (see Fig. 4). However, by comparing the trend of the theoretical CTLs for both TMs, and specially the double donor levels, it is clear that the $\mathrm{E}(+/++)$ level for Ti was correctly assigned to the DLTS peak H180, with activation energies within $0.25-0.32 \mathrm{eV}$. Since TMs impurities in silicon are known to interact with lighter foreign elements (such as oxygen, hydrogen...), it has been hypothesized in [11] that the origin of the intermediate band gap reported by $[6,7,9]$ is actually a hydrogen-titanium complex. First efforts to support this evidence by the DFT community $[12,14]$ have been made; however, the large number of possible structural configurations for such $\mathrm{Ti}-\mathrm{H}$ complexes does not allow for a one-to-one assignment. 


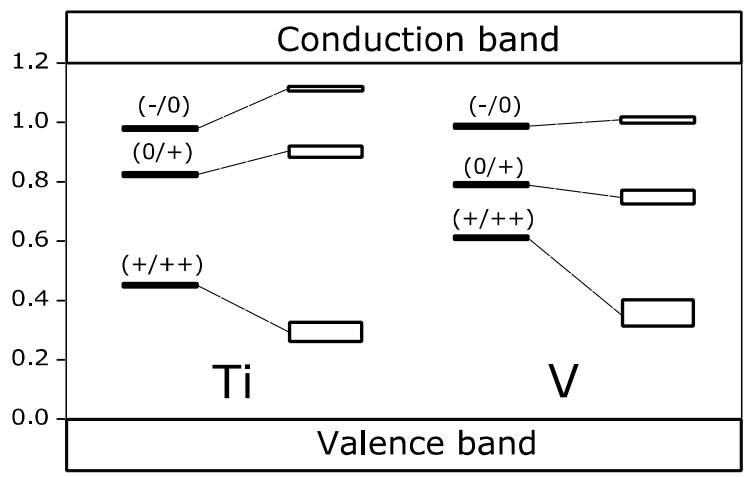

Fig. 4. Charge Transitions Levels (CTLs) for interstitial Ti and V computed with the DFT-GW combine approach (black lines) and measured DLTS activation energies [6-11,20,21] (white rectangles). The width of the white rectangles represents the dispersion of the reported values.

We report a single acceptor, (-/0), a single donor, (0/+), and a double donor, $(+/++)$, levels for both $\mathrm{Ti}$ and $\mathrm{V}$, by using state-of-the art first-principles and parameter free approaches, namely DFT $+\mathrm{GW}$. The computed single levels are in fair agreement (within 0.1-0.15 eV) with the experimental ones (see Fig. 4). Similar agreements have been reported by [26] for a series of point defects in several types of semiconductors. The reported double donor levels, on the other hand, lay a bit further from the experimental references (within 0.15-0.25 eV). The comparison of such absolute error with previous reported double levels in the GW framework is not straight forward since such CTLs usually lay within the bulk valence band for the majority of systems and therefore they are invisible to DLTS measurements.

\section{CONCLUSIONS}

Atomic scale calculations accurately predict point defect properties, since we were able to validate our results with experimental evidence (EPR and DLTS studies). The DFTGW combine approach is the state-of-the art first-principles method to compute CTLs in semiconductors, giving values in fair agreement with the DLTS measurements, without including empirical shifts or fitted parameters. The discussed approach is still limited to small set of systems due to the large number of unoccupied states needed to converge the GW calculation.

The presented work can be considered as a first step into the evaluation of electrical activity of TMs in silicon. Further investigations should include meaningful calculations of the probability of capturing/releasing charges by the already computed CTLs.

\section{REFERENCES}

[1] H. H. Woodbury and G. W. Ludwig, "Spin resonance of transition metals in silicon", Phys. Rev., vol. 117,pp. 102, 1960.

[2] G. W. Ludwig and H. H. Woodbury, "Solid States Physics" edited by F. Seitz and D. Turnbull, Vol. 13 (Academic Press, New York, 1962).

[3] F. Beeler, O. K. Anderson, and M. Scheffler, "Theoretical evidences for low-spin ground states of early interstitial and late substitutional 3d transtion-metal ions in silicon", Phys. Rev. Lett., vol. 55, pp. 1498, 1985
[4] D. A. van Wezep, R. van Kemp, E. G. Sieverts and C. A. J. Ammerlaan, "Electron-nuclear double resonance of titanium in silicon: ${ }^{29}$ Si ENDOR", Phys. Rev. B, vol 32, pp. 7129, 1985.

[5] D. A. van Wezep and C, A, J, Ammerlaan, "Electron-nuclear double resonance of titanium in silicon: ${ }^{47} \mathrm{Ti}$ and ${ }^{49} \mathrm{Ti}$ ENDOR", Phys. Rev. B, vol. 37 pp. 7268,1988 .

[6] J. W. Chen, A. G. Milnes and A. Rohatgi, "Titanium in silicon as a deep level impurity”, Solid State Elect., vol. 22, pp. 801-808, 1979.

[7] J. R. Morante, J. E. Carceller, P. Cartujo and J. Barbolla, "Thermal emision rates and capture cross-section of majority carriers at titanium levels in silicon", Solid State Elect., vol. 26, pp. 1-6, 1983.

[8] A. C. Wang and C. T. Sah, "Complete electrical characterization of recombination propeties of titanium in silicon", J. Appl. Phys., vol. 56, pp. 1021, 1984.

[9] Z. Shurong, L. Yongling and F. Chunyin, "Several physical properties of titanium in silicon", J. of Electronics, vol. 5, pp. 40-46, 1988.

[10] D. Mathiot and S. Hocine, " Titanium-related deep levels in silicon: a reexamination”, J. Appl. Phys., vol. 66, pp. 5862, 1989.

[11] L. Scheffler, VI. Koljovsky and J. Weberm "Identification of titanium-hydrogen complexes with up to four hydrogen atoms in silicon”, J. Appl. Phys., vol. 117, pp. 085707, 2015.

[12] D. J. Backlund and S. K. Estreicher, "Structural, electrical, and vibrational properties of Ti-H and Ni-H complexes in Si”, Phys. Rev. B vol.81 pp. 155208, 2010.

[13] V. P. Markevich, S. Leonard, A. R. Peaker, B. Halmiton, A. G. Marinopoulos and J. Coutinho, "Titanium in silicon: lattice positions and electronic properties", Appl. Phys. Lett., vol. 104, pp. 152105, 2014.

[14] P. Santos, J. Coutinho, V. J. B. Torres, M. J. Rayson and P. R. Briddon, "Hydrogen passivation of titanium impurities in silicon: effect of doping conditions", Appl. Phys. Lett., vol. 105, pp. 032108, 2014.

[15] A. G. Marianopoulos, P. Santos and J. Coutinho, "DFT+U study of electrical levels and migration barriers of early $3 \mathrm{~d}$ and $4 \mathrm{~d}$ transition metals in silicon", Phys. Rev. B, vol. 92, pp. 075124, 2015.

[16] P. Rinke, A. Janotti, M. Scheffler and C. G. Van de Walle, "Defect formation energies without the band-gap problem: combining densityfuncation theory and the GW approach for the silicon self-interstitial", Phys. Rev. Lett., vol. 102, pp. 026402, 2009.

[17] X. Gonze et al., "First-principles computation of material properties: the ABINIT software project", Comp. Mat. Sci., vol. 25, pp. 478-492, 2002.

[18] D. R. Hamann, "Optimized norm-conserving Vanderbilt pseudopotentials", Phys. Rev. B, vol. 88, pp. 085117, 2013.

[19] J. P. Perdew, K. Burke and M. Ernzerhof, "Generalized gradiente approximation made simple", Phys. Rev. Lett., vol. 77, pp. 3865, 1996.

[20] K. Graff in "Metals Impurities in silicon-device fabrication", edited by R. Hull, R. N. Osgood, H. Sakaku and R. J. Kriegler and Q. Zunger, Springer, 1981.

[21] D. J. Backlund, T. M. Gibbons, and S. K. Estreicher, "Vanadium interactions in crystalline silicon", Phys. Rev. B, vol. 94, pp.195210, 2016.

[22] J. Coutinho, V. B. Torres, R. Jones and P. R. Briddon, "Electrical activity of chalcogen-hydrogen defects in silicon", Phys. Rev. B, vol. 67 ,pp. 035205, 2003

[23] M. Jain, J. R. Chelikowsky and S. G. Louie, "Quasiparticle excitations and charge transition levels of oxygen vacancies in hafnia”, Phys. Rev. Lett., vol. 107, pp. 216803, 2011.

[24] G. Makov and M. C. Payne, "Periodic boundary conditions in ab initio calculations", Phys. Rev. B, vol. 51, pp. 4014, 1995.

[25] C. Freysoldt, B. Grabowski, T. Hickel, J. Neugebauer, G. Kresse, A. Janotti and C. G. Van de Walle, "First-principles calculations for point defects in solids", Rev. Mod. Phys., vol. 86, pp. 253, 2014.

[26] W. Chen and A. Pasquarello, "Accuracy of GW for calculating defect energy levels in solids", W. Chen and A. Pasquarello, Phys. Rev. B, vol. 96, pp. 020101, 2017. 